\title{
Universal dependence of the fluctuation-dissipation ratio on the transition rates in trap models
}

\author{
F Ritort * \\ Department of Physics, Faculty of Physics, University of Barcelona \\ Diagonal 647, 08028 Barcelona, Spain \\ ritort@ffn.ub.es
}

June 8,2018

\begin{abstract}
We investigate violations of the fluctuation-dissipation theorem in two classes of trap models by studying the influence of the perturbing field on the transition rates. We show that for perturbed rates depending upon the value of the observable at the arrival trap, a limiting value of the fluctuation-dissipation ratio does exist. However, the mechanism behind the emergence of this value is different in both classes of models. In particular, for an entropically governed dynamics (where the perturbing field shifts the relative population of traps according to the value of the observable) perturbed rates are argued to take a form that guarantees the existence of a limiting value for the effective temperature, utterly related to the exponential character of the distribution of trap energies. Fluctuationdissipation (FD) plots reproduce some of the patterns found in a broad class of glassy systems, reinforcing the idea that structural glasses self-generate a dynamical measure that is captured by phenomenological trap models.
\end{abstract}

\section{Introduction}

Glasses are widespread materials that yet resist a basic comprehension from many points of view. Although several aspects concerning their macroscopic behavior have been modeled, showing qualitative agreement with experimental results, still our understanding of the relevant physics at the mesoscopic scale remains largely speculative. Long timescales (i.e. several orders of magnitude larger than the microscopic timescales) associated to physical processes occurring at very small length scales (of the order of several nanometers) are thought to be common. The main difficulty behind a theoretical description of glassy dynamics is caused by the great disparity between timescales and length scales, where neither hydrodynamic approaches or standard nucleation theories can be applied. It is for this reason that trying to understand the dynamical behavior of glasses remains a theoretical challenge. Its comprehension may open new routes to the understanding of a large class of new physical phenomena, where such a strong disparity of temporal and spatial scales is relevant.

The glass transition problem has been studied from many perspectives. In the absence of a well established basic microscopic theory modeling offers many possibilities. A

\footnotetext{
* On leave at Department of Physics, University of California, Berkeley CA 94720, USA
} 
successful category of models for the glass transition are trap models [1, 2]. They have the advantage of capturing some of the relevant features of glassy relaxation within a tractable formalism. Trap models have been widely investigated (for a review, see [3]) and found to reproduce pretty well several experimental features such as the decay of the thermoremanent magnetization in spin glasses [4, 13, or the super-activated behavior of the viscosity in glasses [5]. Glassy systems are characterized by aging and violations of the fluctuation-dissipation theorem (FDT) (for recent reviews see [6, 7]). FDT violations can be quantified by defining the fluctuation-dissipation ratio (FDR),

$$
X(t, s)=\frac{T R(t, s)}{\frac{\partial C(t, s)}{\partial s}}, \quad t>s
$$

where $T$ is the temperature of the bath (the Boltzmann constant $k_{B}$ is set to 1 ) and $C(t, s), R(t, s)$ are the two-times correlation and response functions. The FDR allows to define also the effective temperature,

$$
T_{\text {eff }}(t, s)=T / X(t, s) \quad .
$$

In equilibrium FDT is satisfied so $X=1$ and $T_{\text {eff }}(t, s)=T$. These definitions would be devoid of any interest if it were not for the fact that a broad category of glassy systems (those exhibiting a one-step replica-symmetry breaking transition in their corresponding mean-field theory) show, in their long-time regime, that $X(t, s) \rightarrow \hat{X}(s)$, i.e. the FDR converges to a non-trivial function of the lowest time $s$. More precisely, these systems show the existence of two-timescales depending upon the value of the ratio $(t-s) / s$ in the asymptotic regime $t, s>>1$. If $(t-s) / s<<1$ then $X(t, s)=1$ and the systems is locally equilibrated (short-time sector). However, if $(t-s) / s \sim \mathcal{O}(1)$ then $X(t, s) \rightarrow \hat{X}(s)$ (long-time sector) being independent on the precise value of the ratio $t / s$ (provided it is finite). Hereafter, we will refer to this category of models as one-step (1S) models as they show a single jump of the value of $X(t, s)$ from 1 to a different value (generally smaller than 1). The existence of $\hat{X}(s)$ shows that the aging state at time $s$ can be characterized by a non-equilibrium parameter that could be identified as the fingerprint of a non-equilibrium temperature, $T_{\text {eff }}(s)=T / \hat{X}(s)$. From a theoretical point of view, the conditions necessary for the existence of $\hat{X}(s)$ in the long-time sector have not been found in full generality. These conditions could be quite strong, though, and the possibility has been raised that only the limiting value $X_{\infty}(s)=\lim _{t \rightarrow \infty} X(t, s)$ (rather than $\hat{X}(s)$ ) is physically meaningful ${ }^{1}$. Most of the numerical studies done on structural glass models seem to be in qualitative agreement with the $1 \mathrm{~S}$ scenario [6].

Usually, FD violations are schematically depicted in the form of FD plots where the integrated-response function $\chi(t, s)=\int_{s}^{t} R\left(t, t^{\prime}\right) d t^{\prime}$ is plotted as a function of $C(t, s)$. For $1 \mathrm{~S}$ models FD plots are made out of two linear segments, one of slope $-1 / T$ covering the short-time sector, the other with slope $-\hat{X}(s) / T$ in the long-time sector.

Recently, a debate has emerged whether trap models can reproduce the pattern characteristic of FD violations in $1 \mathrm{~S}$ systems. In particular, because dynamics within traps is usually not incorporated into the models, they should only reproduce the long-time sector where $\hat{X}(s)$ describes relaxational jumps among traps. Yet, the most recent results on this issue [11] seem to disprove the existence of such $1 \mathrm{~S}$ features.

The goal of this paper is to clarify this issue by showing that FD plots in trap models strongly depend upon the functional dependence of the transition rates on the field. Actually, perfectly straight $1 \mathrm{~S}$ FD plots can be obtained provided the escape rate from a trap

\footnotetext{
${ }^{1}$ The statement has been done in the context of ferromagnetic models at criticality [8] where the universal FDR is $X_{\infty}=\lim _{s \rightarrow \infty} X_{\infty}(s)$. Several analytical works support the conjecture 9] 10]. The conjecture can be naturally extended beyond criticality to generic glassy systems by proposing that $X_{\infty}(s)$ is waiting-time dependent.
} 
is unchanged (within the linear-response regime) by the action of a external perturbation. The study of two different classes of trap models shows the existence of a value $X_{\infty}(s)$ for the FDR (1) in the limit $t \rightarrow \infty$, yet the mechanism behind the emergence of this limiting value is different. For a dynamics governed by entropic effects there exists an effective temperature $T_{\infty}(s)$ that remains finite in the limit $T \rightarrow 0$ and is uniquely determined by the width of the exponential distribution of trap energies. This shows that $1 \mathrm{~S}$ behavior with $X_{\infty}(s)$ proportional to $T$ at low $T$ is the fingerprint of a slow dynamics governed by entropic rather than thermally activated effects.

\section{Trap models: some definitions and useful expres- sions}

Following 1 we consider $N$ independent traps labeled by the letter $\alpha$, each trap characterized by an energy $E_{\alpha}$ and a generic observable that we will denote by $M_{\alpha}$. We consider a Markov dynamics among traps. To set the formalism as clear as possible we will consider time as discrete and define a dynamical history by the temporal series $\{\alpha(t)\}$ where $t$ is an integer (and consecutive time steps are separated by $\Delta t=1$ ). The dynamical evolution is specified by the probability $P_{t}(\alpha)$ that the system stays in trap $\alpha$ at time $t$, and by the conditional probability $W\left(\alpha^{\prime} \mid \alpha\right)$ that the system goes from trap $\alpha$ to $\alpha^{\prime}$ in a single time step. We assume conditional probabilities to be time-independent. Eventually we will be interested in the continuous-time limit $\Delta t \rightarrow 0$ where conditional probabilities for $\alpha \neq \alpha^{\prime}$ are vanishingly small $W\left(\alpha^{\prime} \mid \alpha\right) \rightarrow \hat{W}\left(\alpha^{\prime} \mid \alpha\right) \Delta t, \hat{W}\left(\alpha^{\prime} \mid \alpha\right)$ being the transition rates. In what follows we will coin the term rate for $W$ although properly speaking this term should be only adopted for its continuous-time analogue $\hat{W}$. A master equation governs the dynamical evolution of the system,

$$
P_{t}(\alpha)=\sum_{\alpha^{\prime}} P_{t-1}\left(\alpha^{\prime}\right) W\left(\alpha \mid \alpha^{\prime}\right) .
$$

The $W$ will consist of two terms, the term $W\left(\alpha^{\prime} \mid \alpha\right)=A\left(\alpha^{\prime} \mid \alpha\right)$ for $\alpha \neq \alpha^{\prime}$ describing the jumping rate from $\alpha$ to $\alpha^{\prime}$ and the term $W(\alpha \mid \alpha)=B(\alpha)$ describing the rate of staying in trap $\alpha$. Therefore we can write,

$$
W\left(\alpha^{\prime} \mid \alpha\right)=A\left(\alpha^{\prime} \mid \alpha\right)\left(1-\delta_{\alpha, \alpha^{\prime}}\right)+B(\alpha) \delta_{\alpha, \alpha^{\prime}}
$$

Transition rates are normalized $\sum_{\alpha} W\left(\alpha \mid \alpha^{\prime}\right)=1$ and satisfy,

$$
\sum_{\alpha^{\prime} \neq \alpha} A\left(\alpha^{\prime} \mid \alpha\right)+B(\alpha)=1, \quad \forall \alpha \quad .
$$

The transition rates allow to define the escape rate $W_{\text {esc }}(\alpha)$ from a given trap $\alpha$,

$$
W^{\mathrm{esc}}(\alpha)=\sum_{\alpha^{\prime} \neq \alpha} A\left(\alpha^{\prime} \mid \alpha\right)=1-B(\alpha)
$$

Dynamics in trap models is specified once the rates $W$ are given. For the time being we concentrate only in the zero-field case where dynamics is only governed by the energies of the traps. We consider two representative cases: the Bouchaud model (BM) [1] where the rate $A\left(\alpha^{\prime} \mid \alpha\right)$ only depends on the energy $E_{\alpha}$ of the departure trap $\alpha$, and the BarratMezard model (BMM) 2] where the rate $A\left(\alpha^{\prime} \mid \alpha\right)$ depends on both energies of the departure and arrival traps, $E_{\alpha}$ and $E_{\alpha^{\prime}}$. Both models consider an spectrum of traps with energies 
$E_{\alpha} \leq 0$ and are defined by their respective rates,

$$
\begin{array}{r}
A^{\mathrm{BM}}\left(\alpha^{\prime} \mid \alpha\right)=\frac{\omega_{0}}{N} \exp \left(\beta E_{\alpha}\right) \\
A^{\mathrm{BMM}}\left(\alpha^{\prime} \mid \alpha\right)=\frac{\omega_{0}}{N} \frac{1}{1+\exp \left(\beta\left(E_{\alpha^{\prime}}-E_{\alpha}\right)\right)}
\end{array}
$$

where $\beta=1 / T$ and $\omega_{0}$ is a microscopic frequency that we will take inversely proportional to the discretization time $\Delta t$. The choices (78) are justified by the requirement that the detailed balance property is satisfied. The corresponding expressions for $B(\alpha)$ are determined by the normalization condition (5). The energy spectrum of the ensemble of traps is defined by,

$$
\rho(E)=\frac{1}{N} \sum_{\alpha=1}^{N} \delta\left(E-E_{\alpha}\right)
$$

Both models consider an spectrum of states with exponentially distributed energies,

$$
\rho(E)=\frac{1}{T_{g}} \exp \left(\frac{E}{T_{g}}\right) \quad E \leq 0 \quad .
$$

There is an important difference between the dynamics in the BM and the BMM in the low $T$ limit. While dynamics is completely arrested in the BM (the escape rate (6) vanishes exponentially fast with $1 / T$ ) it does not in the BMM where the escape rate is finite even at $T=0^{2}$. This makes the BM model more suitable to describe glassy relaxation in a landscape of traps separated by energy barriers while the BMM is better suited to describe relaxation over entropy barriers.

In the presence of an external field $h$ coupled to the observable $M_{\alpha}$ the rates (78) get modified. The simplest possibility is to assume that the functional form of the rates (178) remains unchanged while replacing

$$
E_{\alpha} \rightarrow E_{\alpha}-h M_{\alpha}
$$

We will refer to this choice as the purely activated rates. A priori, however, other choices maybe equally valid. For instance, let us consider the following rates in the presence of a field ${ }^{3}$,

$$
A_{h}\left(\alpha^{\prime} \mid \alpha\right)=A\left(\alpha^{\prime} \mid \alpha\right) \exp \left(-\beta h\left(\mu M_{\alpha}-\gamma M_{\alpha^{\prime}}\right)\right)
$$

where $\mu$ and $\gamma$ are arbitrary parameters. In this form these rates generally violate detailed balance. Although this choice may seem unjustified, violation of detailed balance may be a necessary ingredient for trap models to reproduce non-equilibrium aspects of glassy systems ${ }^{4}$.

The rates (12) were considered in 4] in the case where $\gamma=1-\mu$ and detailed balance is preserved. However, the implications and physical meaning of the term $\gamma M_{\alpha^{\prime}}$ in (12)

\footnotetext{
${ }^{2}$ This difference between both models appears explicitly in the time decay of the energy in the glassy phase $T<T_{g}$ where the Gibbs energy distribution ceases to be normalizable. While in the BM the energy decays as $E(t) \sim-T \log (t)$ (therefore relaxation arrests at $T=0$ ), in the BBM it decays like $E(t) \sim-T_{g} \log (t)$ for $T \rightarrow 0$ being $T$ independent in that limit.

${ }^{3}$ From now we will use the subscript $h$ to refer to field-dependent rates. For zero-field rates we will drop off the subscript $h$.

${ }^{4}$ Actually, trap models can be justified only as a coarse-grained description where the original phase space is partitioned into components or regions, each of these regions corresponding to a trap (for a discussion of this aspect see Secs. 3.3 and 4.2 in [6]). However, at difference with the original microscopic dynamics, the projected dynamics among traps can violate detailed balance (a property that must be satisfied by the microscopic dynamics for the system to equilibrate).
} 
went unnoticed. Indeed, the choice of purely activated rates $(\mu=1, \gamma=0)$ has occupied the majority of studies of FDT violations in trap models [4, 11, 13. Here we go beyond and show that the existence of the term $\gamma M_{\alpha^{\prime}}$ is crucial for the emergence of $1 \mathrm{~S}$ behavior in FD plots. We will give also a physical interpretation of this term in a relaxational scenario governed by activated and entropic barriers. Our study will consider both the BM and the BMM.

Before delving into each of the two class of models we present some useful formulae needed to derive the FDR. Some of these computations have been addressed in previous works 4, 11, 13, here we reproduce them in more detail. We are interested in computing the response function $R(t, s)$ and express it in terms of derivatives of the correlation function $C(t, s)$. They are defined by,

$$
C(t, s)=<M(t) M(s)>\quad ; \quad R(t, s)=\frac{1}{\Delta t} \frac{\delta<M(t)>}{\delta h(s)} \theta(t-s),
$$

i.e. the response function is the change of the expectation value $\langle M(t)\rangle$ when a impulse field coupled to $M$ is applied at a previous time $s$. For simplicity we will assume that $C(t, t)$ is normalized to unity. If $W_{h}\left(\alpha^{\prime} \mid \alpha\right)$ stands for the rate in the presence of a field then we have,

$$
R(t, s)=\frac{1}{\Delta t} \sum_{\alpha_{t}, \ldots, \alpha_{s}} P_{s}\left(\alpha_{s}\right) M_{\alpha_{t}} \prod_{r=s+1}^{t-1} W\left(\alpha_{r+1} \mid \alpha_{r}\right) \Delta_{h} W\left(\alpha_{s+1} \mid \alpha_{s}\right)
$$

where

$$
\Delta_{h} W\left(\alpha^{\prime} \mid \alpha\right)=\lim _{h \rightarrow 0} \frac{W_{h}\left(\alpha^{\prime} \mid \alpha\right)-W\left(\alpha^{\prime} \mid \alpha\right)}{h}
$$

Using (415) we can rewrite (15) as follows,

$$
\Delta_{h} W\left(\alpha^{\prime} \mid \alpha\right)=a\left(\alpha^{\prime} \mid \alpha\right)-\left[\sum_{\alpha^{\prime \prime}} a\left(\alpha^{\prime \prime} \mid \alpha\right)\right] \delta_{\alpha^{\prime}, \alpha}
$$

with the definition,

$$
a\left(\alpha^{\prime} \mid \alpha\right)=\left(\frac{\partial A_{h}\left(\alpha^{\prime} \mid \alpha\right)}{\partial h}\right)_{h=0} .
$$

The next sections describe how to compute $a\left(\alpha^{\prime} \mid \alpha\right)$ in both the BM and BMM and how an expression for $R(t, s)$ in (14) can be obtained.

Another important concept related to the measured observables $M_{\alpha}$ is neutrality [11, 6]. The probability density of being at time $t$ in a trap with a value $M$ of the observable, conditioned by its energy to be equal to $E$, is given by,

$$
P_{t}(M \mid E)=\frac{\sum_{\alpha} P_{t}(\alpha) \delta\left(M-M_{\alpha}\right) \delta\left(E-E_{\alpha}\right)}{\sum_{\alpha} P_{t}(\alpha) \delta\left(E-E_{\alpha}\right)} .
$$

$M$ is said to be neutral if, assuming that $P_{t=0}(M \mid E)=P_{t=0}(-M \mid E)$, this property stays valid at all times $P_{t}(M \mid E)=P_{t}(-M \mid E)$. As stated, this condition of neutrality is quite stringent. It can be relaxed provided some conditions are met. For sake of simplicity and to successfully convey the main message of this paper, we will assume that the observable density $g(M)$ is symmetric ${ }^{5}$,

$$
g(M)=\frac{1}{N} \sum_{\alpha=1}^{N} \delta\left(M-M_{\alpha}\right) \equiv g(-M)
$$

\footnotetext{
${ }^{5} \mathrm{~A}$ common choice is a Gaussian distribution of zero mean and unit variance such that $C(t, t)=1$ holds.
} 
Eq. (19) together with the initial condition $P_{t=0}(M \mid E)=P_{t=0}(-M \mid E)$ is enough to guarantee neutrality. Neutrality allows to derive a simple relation for the lowest-time derivative of the correlation function $C(t, s)$,

$$
\frac{\partial C(t, s)}{\partial s}=\frac{1}{\Delta t} \sum_{\alpha_{t}, ., \alpha_{s}} P_{s}\left(\alpha_{s}\right) M_{\alpha_{t}} \prod_{r=s}^{t-1} W\left(\alpha_{r+1} \mid \alpha_{r}\right)\left(M_{\alpha_{s+1}}-M_{\alpha_{s}}\right)
$$

If we replace (40) for $W\left(\alpha_{s+1} \mid \alpha_{s}\right)$ in (20) then neutrality implies,

$$
\sum_{\alpha_{s}} P_{s}\left(\alpha_{s}\right) A\left(\alpha_{s}\right) M_{\alpha_{s}}=0
$$

because, for a fixed value of the energy, an identical number of traps contribute to (21) with positive and negative values of $M$. Therefore,

$$
\frac{\partial C(t, s)}{\partial s}=\frac{1}{\Delta t} \sum_{\alpha_{t}, ., \alpha_{s}} P_{s}\left(\alpha_{s}\right) M_{\alpha_{t}} \prod_{r=s+1}^{t-1} W\left(\alpha_{r+1} \mid \alpha_{r}\right) A\left(\alpha_{s}\right) M_{\alpha_{s+1}} .
$$

\section{The Bouchaud model (BM)}

In the BM the unperturbed rates $A^{\mathrm{BM}}\left(\alpha^{\prime} \mid \alpha\right)$ are given by (7) and depend only on the energy of the departure trap so we will adopt the notation $A^{\mathrm{BM}}\left(\alpha^{\prime} \mid \alpha\right) \equiv A^{\mathrm{BM}}(\alpha)$. Let us consider the rates (12),

$$
A_{h}^{\mathrm{BM}}\left(\alpha^{\prime} \mid \alpha\right)=A^{\mathrm{BM}}(\alpha) \exp \left(-\beta h\left(\mu M_{\alpha}-\gamma M_{\alpha^{\prime}}\right)\right)
$$

It is easy to verify that in this case $a\left(\alpha^{\prime} \mid \alpha\right)$ is given by,

$$
a^{B M}\left(\alpha^{\prime} \mid \alpha\right)=-\beta A^{B M}(\alpha)\left(\mu M_{\alpha}-\gamma M_{\alpha^{\prime}}\right)
$$

where $\mu$ and $\gamma$ are two constants which we will assume can take any value (positive or negative). The parameter $\gamma$ appearing in the rates (24) only depends upon the value of the observable $M$ at the arrival trap. At linear order in $h$, the change of the escape rate $W^{\text {esc }}(\alpha)$ (6) is given by

$$
W_{h}^{\mathrm{esc}}(\alpha)-W^{\mathrm{esc}}(\alpha) \sim-\mu \beta h N A^{\mathrm{BM}}(\alpha) M_{\alpha}+\mathcal{O}\left(h^{2}\right)=-\omega_{0} \mu \beta h M_{\alpha} \exp \left(\beta E_{\alpha}\right)+\mathcal{O}\left(h^{2}\right)
$$

and does not depend on $\gamma$. Therefore, while the parameter $\mu$ directly modifies the lifetime of a trap, the parameter $\gamma$ does not modify it (up to linear order in the perturbation). Simply put, while the parameter $\mu$ directly affects the height of the energetic barrier to be surmounted (through thermal activation) to escape from a trap, the parameter $\gamma$ selectively enhances the rate toward the destination trap through a different activated mechanism. In Sec. 5 we present a physically motivated explanation for this dependence.

Note that only if $\gamma=1-\mu$ detailed balance is preserved in (24). Inserting (24) in (1617) we get,

$$
\Delta_{h} W\left(\alpha^{\prime} \mid \alpha\right)=-\beta A^{\mathrm{BM}}(\alpha)\left[\left(\mu M_{\alpha}-\gamma M_{\alpha^{\prime}}\right)-N\left(\mu M_{\alpha}-\gamma \bar{M}\right) \delta_{\alpha, \alpha^{\prime}}\right]
$$

where $\bar{M}=(1 / N) \sum_{\alpha} M_{\alpha}$ is the average magnetization of traps. The following mathematical steps are followed. We insert (26) in (14) and use the identity (4). If the observable $M_{\alpha}$ is neutral then from (19) we have $\bar{M}=0$. Using (22) we get,

$$
R(t, s)=-\beta\left[\mu \frac{\partial C(t, s)}{\partial t}-\gamma \frac{\partial C(t, s)}{\partial s}\right]
$$


where the continuous limit $\Delta t \rightarrow 0$ has been taken ${ }^{6}$. Replacing in (11) we get for the FDR,

$$
X(t, s)=\gamma-\mu\left(\frac{\frac{\partial C(t, s)}{\partial t}}{\frac{\partial C(t, s)}{\partial s}}\right)
$$

Several comments are in order. First, the present analysis covers regimes where detailed balance is violated. We already justified this choice (see footnote 4). In fact, in a timetranslational invariant regime, $X=\gamma+\mu$ which is generally different from 1 . Only if detailed balance holds $(\gamma=1-\mu)$ then $X=1$. Second, $X$ is finite in the $T \rightarrow 0$ limit. In other words, if a physically meaningful effective temperature (2) exists, then it has to be proportional to $T$ and vanish at $T=0$. Let us consider the following aging form of $C(t, s)$,

$$
C(t, s)=\int_{0}^{\infty} d \lambda w(\lambda) \hat{C}_{\lambda}\left(\frac{t-s}{s^{\lambda}}\right) \quad ; \quad \int_{0}^{\infty} d \lambda w(\lambda)=1
$$

where $\hat{C}_{\lambda}(0)=1, \hat{C}_{\lambda}(\infty)=0$. Provided $w(\lambda \rightarrow 0) \rightarrow 0$ then it can be easily proved that for $t \gg s$,

$$
X(t, s) \rightarrow \gamma-\mathcal{O}\left(\frac{s}{t}\right)
$$

and therefore

$$
X_{\infty}=\lim _{t \rightarrow \infty} X(t, s)=\gamma
$$

i.e. the FDR asymptotically converges to a non-trivial quantity $(\gamma)$. The main conclusion of this simple calculation is that for rates given by (24) there is a universal dependence of the FDR (in its long-time limit) on the parameter $\gamma$ describing how transition rates are perturbed at the linear response level (i.e up to linear order in $h$ ). Note that for $\mu=0$, $X(t, s)=\gamma$ (28), the FD plots are full straight lines as in the 1S scenario and the escape rates are unmodified at the linear order in the field (25).

The validity of (30) relies on the fact that the system is aging. Were the system in a stationary state then trivially $X(t, s)=\gamma+\mu$ for all times $t, s$. Therefore, for stationary non-equilibrium systems $X_{\infty}=\gamma+\mu$ and an additional dependence of the limiting value $X_{\infty}$ on $\mu$ is expected.

In general, for models with full aging $C(t, s)=\hat{C}(t / s), X(t, s)$ in (28) is a single function of $C$ yielding limiting FD plots [4, 11]. For instance, the BM is characterized by a non-normalizable distribution for $T<T_{g}$ where the correlation function $C(t, s)$ shows full aging. In Fig. 1 we show FD plots for the BM for a given value of $\gamma$ and different values of $\mu$. Note that for $\mu=0$ a 1S FD plot is obtained characteristic of systems with a single timescale. The purely activated case $\mu=1, \gamma=0$ gives $X_{\infty}=0$ as has been found in [11.

\section{The Barrat-Mezard model (BMM)}

As we already said in Sec. 2 after (10) the main difference between the BM and the BMM is that the escape rates in the later are temperature independent in the low $T$ limit, therefore slow dynamics is governed by entropy barriers. Therefore, the BMM appears as the phenomenological description of the entropic relaxation of several microscopic models such as the Backgammon model [14 or the oscillator model 15].

In this section we want to show that similar conclusions (2513) can also be inferred from the BMM (8) in the low-temperature limit $T \rightarrow 0$ where a purely entropic mechanism

\footnotetext{
${ }^{6}$ Equation (27) was first derived in [4 but for the case where detailed balance holds. Note also that the symmetry condition there expressed (our (19) is not enough for the validity of (27) as full neutrality as expressed by the supplemental condition $P_{t=0}(M \mid E)=P_{t=0}(-M \mid E)$ must hold.
} 


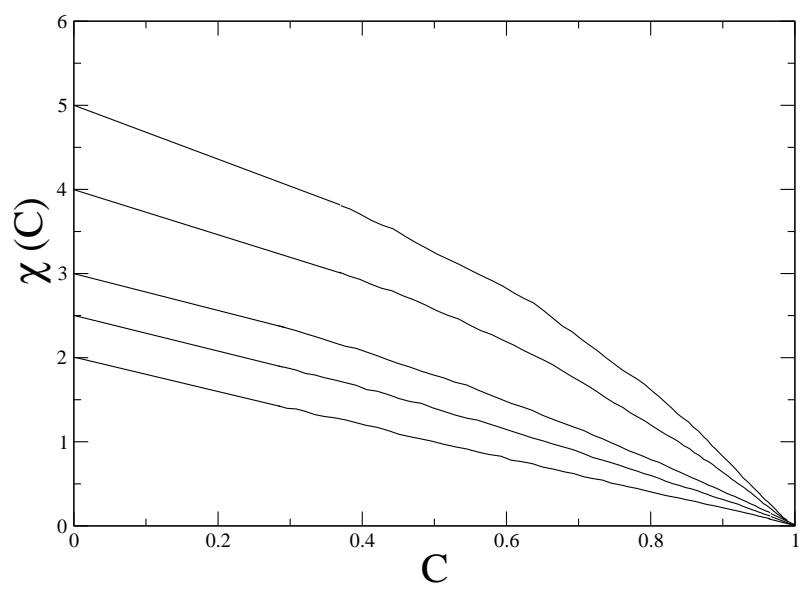

Figure 1: FD plots for the BM at $T=0.5\left(T_{g}=1\right.$ with $g(M)$ a Gaussian distribution of unit variance) at $\gamma=1$ and $\mu=0,0.5,1,2,3$ (from bottom to top). Note that only for $\mu=0$ there is a full straight line, yet in all cases the limiting slope when $C \rightarrow 0$ is the same, $X_{\infty}=\gamma=1$. It can be proved that the value of $\chi(C)$ in the limit $C \rightarrow 0$ is given by $\beta \gamma+\mu / T_{g}$.

of relaxation is at work (in contrast to the BM case where escape rates are thermally activated). The analysis of the FDR in the BMM goes along the same lines as in the preceding section. We start from the unperturbed $T=0$ rates,

$$
A^{\mathrm{BMM}}\left(\alpha^{\prime} \mid \alpha\right)=\frac{\omega_{0}}{N} \theta\left(E_{\alpha}-E_{\alpha^{\prime}}\right)
$$

We remark two aspects of these rates: 1) As these are zero-temperature rates they do not satisfy detailed balance and 2) the rates are independent of the energy of the departure and arrival trap provided that the energy decreases. Nevertheless the escape rate (6) from a given trap will depend on its energy through the relation,

$$
W^{\mathrm{esc}}(\alpha)=\frac{\omega_{0}}{N} \sum_{\alpha^{\prime}} \theta\left(E_{\alpha}-E_{\alpha^{\prime}}\right)=\omega_{0} \int_{-\infty}^{E_{\alpha}} \rho\left(E^{\prime}\right) d E^{\prime}
$$

and will decrease as the energy of the trap $E_{\alpha}$ decreases. Now we need to specify how the rates $W$ change in the presence of a field. Inspired by the corresponding expression for the BM 231) we will assume the following factorized form for the perturbed rates in the $\mathrm{BMM}$

$$
A_{h}^{\mathrm{BMM}}\left(\alpha^{\prime} \mid \alpha\right)=A^{\mathrm{BMM}}\left(\alpha^{\prime} \mid \alpha\right) \exp \left(-h\left(\mu M_{\alpha}-\gamma M_{\alpha^{\prime}}\right)\right) .
$$

where $A_{h}^{\mathrm{BMM}}\left(\alpha^{\prime} \mid \alpha\right)$ is given by (32). This functional dependence assumes that transitions to new traps are enhanced towards larger values of the observable $M_{\alpha^{\prime}}$, however arrival traps must have energies below that of the departure trap $E_{\alpha^{\prime}}<E_{\alpha}$. This form of the perturbed rates does not correspond to the case of purely activated rates where (32) are modified according to the shift (11). In fact, were this the case then there would be correlations between energies and observable values, and transitions with $E_{\alpha^{\prime}}>E_{\alpha}$ would occur depending on the difference $M_{\alpha^{\prime}}-M_{\alpha}$. The factorized form of the rates (34) as well as the enhancement of rates towards traps with larger values of $M_{\alpha}$ are not easy to justify a priori. They have to be seen as an approximate solution to the real perturbed 
rates that takes into account the dependence of the rates upon the observable value taken at the arrival trap ${ }^{7}$.

The enhancement of rates towards traps with larger $M_{\alpha^{\prime}}$ can be explained as follows. Let us assume that $\rho(E)$ is a monotonically increasing function of $E$ (e.g. as in (10)). The main effect of the field on a shell of traps that have a given energy is that the population of traps with $M>0$ tends to increase relative to those with $M<0$. The shift of populations can be computed as follows. Given the unperturbed density $\rho^{(2)}(E, M)=\rho(E) g(M)$ we can write,

$$
\begin{gathered}
\rho_{h}^{(2)}(E, M)=\int_{-\infty}^{0} d E^{\prime} \rho\left(E^{\prime}\right) g(M) \delta\left(E-E^{\prime}-M h\right)=\rho(E+M h) g(M) \\
=\rho(E) g(M)\left(1+\frac{\partial \log (\rho(E))}{\partial E} M h+\mathcal{O}\left(h^{2}\right)\right)=\rho^{(2)}(E, M) \exp (\gamma(E) M h)
\end{gathered}
$$

the final expression being valid up to linear order in $h$ and where we have introduced the function $\gamma(E)$,

$$
\gamma(E)=\frac{\partial \log (\rho(E))}{\partial E}=\frac{\partial S(E)}{\partial E} .
$$

where $S(E)=\log (\rho(E))$ is an entropy an $\gamma$ has the dimensions of the inverse of a temperature. Analogously, if we define an entropy $S^{(2)}(E, M)=\log \left(\rho^{(2)}(E, M)\right)$ then we have, up to linear order in $h, S_{h}^{(2)}(E, M)=S^{(2)}(E, M)+\gamma(E) M h$. The schematic behavior of $S_{h}^{(2)}(E, M)$ is depicted in Fig. 2 For an exponential distribution of states (10) we have,

$$
\gamma(E) \equiv \gamma=\frac{1}{T_{g}}
$$

Although the density $\rho_{h}(E, M)$ changes at linear order in $h$, the symmetry property (19) ensures that the total density $\int_{-\infty}^{\infty} \rho_{h}(E, M) d M$ only changes at the next order $h^{2}$. We stress that the parameter $\gamma$ (expressing the dependence of the perturbed rates upon the value of the observable evaluated at the arrival trap), rather than $\mu$, is the only term describing the entropic unbalance of traps. From (6.34 we obtain for the escape rates, at linear order in the field,

$$
W_{h}^{\mathrm{esc}}(\alpha)=\omega_{0} \exp \left(\frac{E_{\alpha}-h \mu M_{\alpha}}{T_{g}}\right)
$$

where we used the result $\bar{M}=0$ and (33). Calculations identical to those shown for the BM in Sec. 3 lead to,

$$
R(t, s)=-\left[\mu \frac{\partial C(t, s)}{\partial t}-\gamma \frac{\partial C(t, s)}{\partial s}\right]
$$

Note that, contrarily to (27), the response is now finite in the $T=0$ limit, therefore the FDR vanishes linearly with $T$ as $T \rightarrow 0$. Rather than $X$ it is useful to consider the effective temperature (2),

$$
T_{\mathrm{eff}}(t, s)=\left[\gamma-\mu\left(\frac{\frac{\partial C(t, s)}{\partial t}}{\frac{\partial C(t, s)}{\partial s}}\right)\right]^{-1}
$$

\footnotetext{
${ }^{7} \mathrm{~A}$ contribution to the perturbed rates of the form (34) seems to us essential for the emergence of non-trivial effective temperatures. After completion of this work, calculations by Sollich (see note added at the end of the paper) have shown that purely activated rates are equivalent to with $\mu=2 / T_{g}, \gamma=0$ where there is no dependence of the modified rates upon the observable value at the arrival trap.
} 


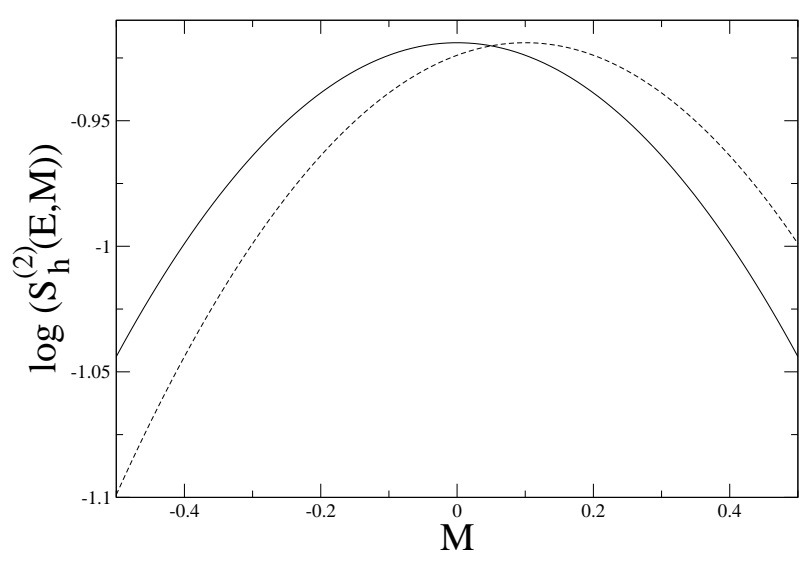

Figure 2: Illustrative picture for $S_{h}^{(2)}(E, M)$ (Gaussian distribution $g(M)$ of unit variance and $T_{g}=1$ ) as a function of $M$ for a fixed value of the energy. $h=0$ (continuous line) and $h=0.1$ (dashed line). The difference between the two curves is linear with $h$ and the slope is equal to $h / T_{g}=0.1$.

Again, under the same assumptions (29) and using (38) we get in the limit $t \rightarrow \infty$,

$$
T_{\infty}(s)=\lim _{t \rightarrow \infty} T_{\text {eff }}(t, s)=\frac{1}{\gamma}=T_{g}
$$

which is independent of $s$ and $\mu$. Therefore, in the limit $t \rightarrow \infty$ the effective temperature coincides with $T_{g}$ which is the width of the exponential distribution of states through (3738). The change of the escape rate (6) induced by the field is given by

$$
W_{h}^{\mathrm{esc}}(\alpha)-W^{\mathrm{esc}}(\alpha)=-\omega_{0} \mu h M_{\alpha} \exp \left(\frac{E_{\alpha}}{T_{g}}\right)+\mathcal{O}\left(h^{2}\right)
$$

and the escape rate, as for the $\mathrm{BM}$, only depends on the value of $\mu$. Again, full straight FD plots are obtained for $\mu=0$ when the escape rates (or trapping times) are unchanged by the field. For the BMM, computations of (4142) can be made explicit due to the simplicity of the expression for the correlation function, $C(t, s)=s / t$ for $t, s \gg 1$ [2]. For the integrated response function we get,

$$
\chi(t, s) \equiv \chi(C)=\int_{s}^{t} d t^{\prime} R\left(t, t^{\prime}\right)=\gamma(1-C)+\frac{\mu}{2}\left(1-C^{2}\right)
$$

and the effective temperature,

$$
\frac{1}{T_{\mathrm{eff}}(C)}=-\frac{\partial \chi(C)}{\partial C}=\gamma+\mu C
$$

giving for $\mu=0$ straight FD plots characteristic of $1 \mathrm{~S}$ behavior, $\chi(C)=\gamma(1-C)$. Note that, in the $\mu=0$ case, $\chi(C=0)=\gamma=1 / T_{g}$ which is the value of the susceptibility at the transition temperature. Fig. [3 shows some typical FD plots.

\subsection{Numerical test of the BMM solution with rates (34).}

To verify the correctness of the previous solution we have done a numerical simulation of the BMM with rates (34). We have considered a Gaussian distribution of observables 


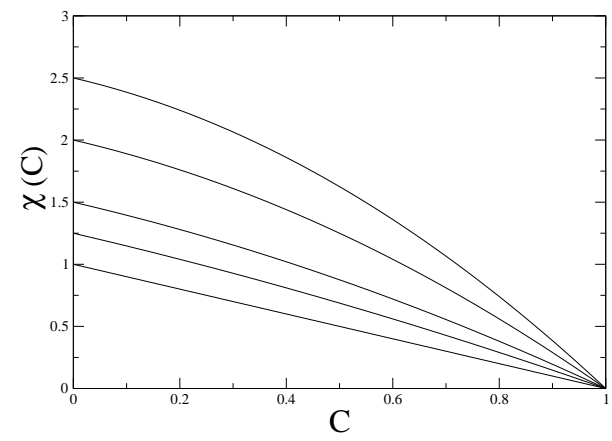

Figure 3: FD plots for the BBM at $T=0\left(T_{g}=1\right.$ with $g(M)$ a Gaussian distribution of unit variance) at $\gamma=1$ and $\mu=0,0.5,1,2,3$ (from bottom to top). Note that only for $\mu=0$ there is a full straight line, yet in all cases the limiting slope when $C \rightarrow 0$ is the same, $T_{\infty}=T_{g}=\gamma=1$.

$g(M)=(2 \pi)^{-1 / 2} \exp \left(-M^{2} / 2\right)$ and $T_{g}=1$. Let $E, M$ be the energy and trap at the departure state. The procedure is as follows: 1) a trap with observable $M^{\prime}$ is chosen with a probability proportional to $\exp \left(M^{\prime} h / T_{g}\right) g\left(M^{\prime}\right)$, i.e. $M^{\prime}=r+h / T_{g}$ where $r$ is a Gaussian (zero mean and unit variance) random number; 2) the lifetime of the trap, according to (39), is given by $\left.\tau=\left(1 / \omega_{0}\right) \exp (-E+M h) ; 3\right)$ The arrival energy is given by $E^{\prime}=E+\log \left(r^{\prime}\right)$ where $r^{\prime}$ is a random number uniformly distributed between 0 and 1 . We start from the disordered initial state $E=0, M=r$ where $r$ is a Gaussian random number as before. We then run two independent simulations by iterating many times the previous steps (one run is at $h=0$ for all times, the other run is at $h=0$ until time $s$ and later a small field $h$ is switched on) and generate correlations and susceptibilities (44) at different times $t, s$. For $t, s>1 / \omega_{0}$ the asymptotic aging solution is obtained as well as the limiting FD plots (44). We verified the correctness of the solution $C(t, s)$ at zero field. The numerical results are shown in Fig. 4 (right) together with the analytical prediction (38) with $\gamma=\mu=1$ (continuous line).

\section{Discussion}

The entropic mechanism described in the preceding section for the BMM provides an explanation on the emergence of an effective temperature given by the width of the exponential density of trap energies ${ }^{8}$. However, is it possible to extend that explanation to the BM where such entropic mechanism appears difficult to reconcile with the explicit activated behavior of the rates (23)? In fact, there is an important difference between the BM and the BMM. While in the later the perturbed rates are not thermally activated (see (34)) leading to a value of $X_{\infty}$ proportional to $T$ at low $T$, in the former the perturbed rates are thermally activated (23) leading to a value of $X_{\infty}$ that is temperature independent at low $T$.

The following explanation, albeit speculative, might be useful. Consider a glass where relaxation is driven by the nucleation of cooperatively rearranging regions. The nucleation

\footnotetext{
${ }^{8}$ In the mean-field theory of spin glasses such origin of the effective temperature has been already remarked [16, 17]. Yet in these models activated processes are completely suppressed.
} 


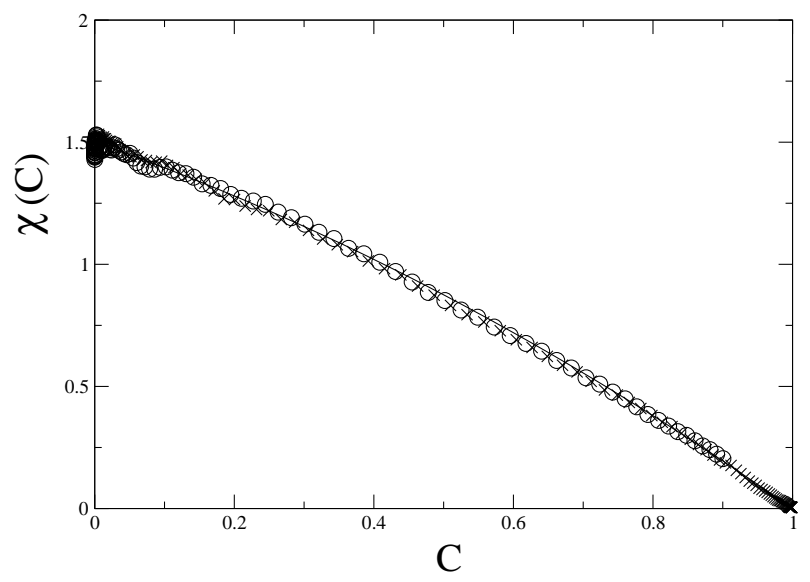

Figure 4: Numerical test of (44). FD plots for the BBM with $\gamma=\mu=T_{g}=1, \omega_{0}=1$ and two waiting times $s=10$ (circles) and $s=1000$ (crosses) in the asymptotic regime $s>>1 / \omega_{0}$. The continuous line is the analytical result (44).

of such a region requires the simultaneous activation of $M$ independent processes, each one characterized by a thermally activated rate $w^{9}$. In the presence of a perturbation of intensity $h$ the rates get modified $w_{h} \sim w \exp (-\beta h \mu)$, where $w$ is the rate at zero field and $\mu$ is a microscopic parameter. The modified rate for the nucleation of the region is $w_{h}^{M} \sim w^{M} \exp (-\beta h \mu M)$. If $\alpha, \alpha^{\prime}$ denote the configurations of the system before nucleating and after nucleating respectively, then $M$ can be identified with the net change of the observable $M_{\alpha}-M_{\alpha^{\prime}}$ after nucleation takes place. The overall rate for that nucleating process is $\exp \left(-\beta h \mu\left(M_{\alpha^{\prime}}-M_{\alpha}\right)\right)$ which coincides with (23) with $\gamma=\mu$.

This scenario could describe diffusive processes (such as low $T$ coarsening) where partial equilibration occurs in the short-time sector corresponding to relaxation within the bulk of domains. In this case, as $X\left(t, s \rightarrow t^{-}\right) \rightarrow \gamma+\mu \rightarrow 1$, the identity $\gamma=\mu$ gives $X_{\infty}=\gamma=\mu=1 / 2$, a result that has been found in the one-dimensional Glauber-Ising chain [20, 9, 12, Let us mention that FD plots similar to those shown here (Figs. 1] 3) with a limiting value $X_{\infty}=1 / 2$ have been reported in one-dimensional models [12, yet it would be interesting to rigorously explain their emergence along the present line of reasoning. The conclusion we draw from this paragraph is that a scenario where $X_{\infty}$ is finite at $T=0$ does not appear compatible with an entropically driven relaxation.

The validity of the expression $w_{h} \sim w \exp (-\beta h \mu)$ requires that all rates corresponding to all elementary activated processes are modified by the same amount (up to linear order in $h$ ) by the perturbation, a requirement that may not be always fulfilled. For instance, let us consider a spin-glass Ising system staying in a locally stable energy minima while aging (i.e. the configuration is stable against all one-spin flips). Assume also that $P$ spins (pointing in different directions) need to be simultaneously activated for the system to escape from that local minima. If there is no long-range order (as is common in spin glasses) the local field distribution is symmetric around zero, so the local fields acting on these $P$ spins will point in different directions. A uniform perturbing field will therefore increase or decrease the local field, and the modified rates $w_{h} \sim w \exp (-\beta h \mu)$ will not be the same for all $P$ spins. Therefore, the mechanism behind the emergence of effective temperatures in frustrated glassy systems (as compared to coarsening systems) seems better

\footnotetext{
${ }^{9}$ Several scenarios based on this cooperative behavior have been proposed 18 19 to explain the origin of super-activated effects in structural glasses.
} 
described by the BMM rather than by the BM.

There are two additional aspects we would like to discuss. One aspect concerns the predicted waiting-time dependence of $X_{\infty}$ and $T_{\text {eff }}$ in $1 \mathrm{~S}$ models. For trap models described by a time-independent density of traps (10) such dependence is not expected, indeed the limiting values (3142) are time independent. For generic glassy systems, though, the width of the exponential density of traps (i.e. $\gamma$ according to (38) should be time dependent as the average energy decreases. Including such dependence would produce FD plots identical to those found in other solvable models like the oscillator model [15]. The second aspect concerns the characteristic form of FD plots in realistic glass models. How to reproduce 1S FD plots with two linear segments, one for $C \rightarrow 1$, the other for $C \rightarrow 0$ ? This requires the introduction of some internal dynamics within traps which is absent in the original formulation of both the BM and the BMM. In the same vein, generalization of this treatment beyond the $1 \mathrm{~S}$ pattern to many timescales could be accomplished through more general transition rates depending on trap correlations $q_{\alpha, \alpha^{\prime}}=M_{\alpha} M_{\alpha^{\prime}}$ in some type of hierarchical structure 4 .

Finally some words about the violation of the property of detailed balance that we have tacitly assumed in the rates 23134). Detailed balance is of course violated in the $\mathrm{BMM}$ at $T=0$. As we have already insisted on (footnote 4 ) this assumption is essential, as trap models are coarse-grained descriptions in phase space of a microscopic dynamics that satisfies that property. The rates $W\left(\alpha^{\prime} \mid \alpha\right)$ in (3) are obtained by projection of the microscopic probabilities and rates onto the corresponding coarse-grained representation of the original phase space. In this projected description detailed balance is not fulfilled anymore (for a discussion see Sec.3.3 in [6]). Moreover, detailed balance is known to be violated in driven systems, yet they also show $1 \mathrm{~S} \mathrm{FD} \mathrm{violating} \mathrm{patterns.} \mathrm{Therefore,} \mathrm{an}$ explanation on the emergence of effective temperatures cannot rely on the fulfillment of the detailed balance property but need to be more general. A coarse-grained description of glassy dynamics, where rates are entropically driven, accomplishes this goal.

\section{Concluding remarks}

Trap models represent a useful approach to understand universal aspects of glassy dynamics. They can be seen as a coarse-grained description of structural relaxation where nucleation of cooperatively rearranging regions is modeled by jumps among traps. They are expected to be relevant to describe relaxation processes in structural glasses. The vast majority of theoretical and numerical studies on glassy systems show that one-step (1S) FD plots are common in the aging regime. However, recent studies on trap models have not found indications of $1 \mathrm{~S}$ behavior in FD plots, casting doubts on the suitability of trap models to reproduce this particular feature of glassy systems.

In this paper we have addressed this issue by showing that proper treatment of the influence of the perturbation on the transition rates partially resolves the problem. Indeed, $1 \mathrm{~S}$ plots can be obtained if entropic effects, driven by the unbalance of the population of traps in the presence of a field, are included in the analysis. We concentrated our attention in both the Bouchaud model (BM) and the Barrat-Mezard model (BMM), in this last case at zero-temperature to better emphasize the main result. We find that $X_{\infty}=\lim _{t \rightarrow \infty} X(t, s)$ (for the BM) and $T_{\infty}=\lim _{t \rightarrow \infty} T_{\text {eff }}(t, s)$ (for the BMM at $T=0$ ) are universal quantities that only depend on a parameter $(\gamma)$ describing the dependence of the perturbed rates on the observable value at the arrival trap. Yet, the physical origin of this dependence appears very different in both models. While perturbed rates are activated in the BM, they are not in the BMM but originate from the entropic unbalance of traps upon the action of the field. This shows that 1S behavior in glassy systems, and the emergence of finite effective temperatures in the low $T$ limit, are the fingerprint of 
a dynamics governed by entropic relaxational processes. Straight 1S plots (compatible with a unique long-time sector) are obtained in both models only when escape rates are unchanged (up to the linear order) by the action of the field.

Which model (BM or BMM) represents more faithfully the slow dynamics of $1 \mathrm{~S}$ systems? The most probable answer is that neither the BM or the BMM provides a complete qualitative description of glassy relaxation and that aspects of both models must simultaneously intertwine in the overall relaxation. The dependence of the perturbed rates on the observable taken at the departure trap (the term including the parameter $\mu$ in (23) can be identified with energy barriers governing the thermally activated escape process from a trap. While the dependence on the observable taken at the arrival trap (the term including the parameter $\gamma$ in (34) is consequence of the unbalance of the number of traps induced by the field, leading to temperature independent entropic relaxation. Also, the full straight-line FD plots obtained for $\mu=0$ represent an extremely simplified behavior of a more realistic scenario where a dependence of the trapping time at linear order in the field is expected. Yet, this dependence is not in conflict with the emergence of "quasi-straight" FD plots for which the existence of a limiting value $T_{\infty}(s)$ is enough.

There remain several open question for the future. It could be instructive to extend this analysis to the BMM at finite temperature. However, a relevant question is to understand how all these considerations are to be generalized to include the case of arbitrary observables. For the non-neutral case, explicit computations as have been done here are not possible. Only for the purely activated case calculations can be done [11, but that analysis does not include the entropic effects discussed in this paper. Yet, there may exist general proofs from which one can deduce specific predictions to be challenged in several microscopic theoretical models and well as in numerical simulations.

Acknowledgments. I warmly thank P. Sollich for communicating me his results after submission of the first draft of the paper and the fruitful exchange of ideas that followed after. FR is supported by the Spanish Ministerio de Ciencia y Tecnología Grant BFM20013525 and Generalitat de Catalunya. FR has benefited from the warming hospitality at the Bustamante lab in the University of California in Berkeley where this work has been done.

Note added: Just after completion of this worked I learned of calculations by Sollich showing that, for the BMM, purely activated rates (11) lead to FD plots not displaying $1 S$ behavior, yet they seem to be described by [34) with $\gamma=0, \mu=2 / T_{g}$, i.e. without dependence of the rates upon the value of the observable taken at the arrival trap. FD plots are therefore very similar to those found in the BM with purely activated rates. This result is very interesting as it shows that the emergence of $1 S$ behavior in trap models cannot be described by only shifting the energies according to the value of the observable [cfr. [11)]. Identification of mechanisms behind the emergence of perturbed rates such as (2334), beyond the purely activated ones, is certainly needed to clarify this issue.

\section{References}

[1] J.-P. Bouchaud, J. Physique I, 2, 1705 (1992)

[2] A. Barrat and M. Mezard, J. Physique I, 5, 941 (1995)

[3] J. P. Bouchaud, L. F. Cugliandolo, J. Kurchan and M. Mezard in Spin Glasses and Random Fields A. P. Young (Ed), World Scientific, Singapore (1998)

[4] J.-P. Bouchaud and D. S. Dean, J. Physique I, 5, 265 (1995)

[5] J.-P. Bouchaud, A. Comtet and C. Monthus, J. Physique I, 5, 1521 (1995); C. Monthus and J.-P. Bouchaud, J. Phys. A (Math. Gen.) 29, 3847 (1996)

[6] A. Crisanti and F. Ritort, Preprint condmat/0212490 to appear in J. Phys. A

[7] L. F. Cugliandolo, Preprint condmat/0210312 
[8] C. Godreche and J. M. Luck, J. Phys. A (Math. Gen.) 33, 1151 (2000)

[9] E. Lippiello and M. Zannetti, Phys. Rev. E 61, 3369 (2000)

[10] P.Calabrese and A. Gambassi, Phys. Rev. E 65, 066120 (2002)

[11] S. M. Fielding and P. Sollich, Phys. Rev. Lett. 88, 050603 (2002)

[12] P. Sollich, S. Fielding and P. Mayer, J. Phys. C (Cond. Matt.), 14, 1683 (2002).

[13] E. Bertin and J.-P. Bouchaud, J. Phys. A (Math. Gen.) 35, 3039 (2002)

[14] F. Ritort, Phys. Rev. Lett., 75, 1190 (1995)

[15] L. L. Bonilla, F. G. Padilla and F. Ritort, Physica A, 250, 315 (1998)

[16] M. Mezard, G. Parisi and M. A. Virasoro, J. Physique. Lett. 46, L217 (1985)

[17] S. Franz and M. A. Virasoro, J. Phys. A (Math. Gen.) 33, 891 (2000)

[18] A. Crisanti and F. Ritort, Preprint condmat/0102104

[19] D. Chakrabarti and B. Bagchi, Preprint condmat/0303153

[20] C. Godreche and J. M. Luck, J. Phys. A (Math. Gen.) 33, 1151 (2000) 